Pacific Journal of Mathematics

ON RANK 3 PROJECTIVE PLANES

JOSEPH KALAHER 


\title{
ON RANK 3 PROJECTIVE PLANES
}

\author{
MichaEL J. KALLAhER
}

One of the unsolved problems in the theory of projective planes is the following: Is every finite projective plane with a transitive collineation group desarguesian? This problem is investigated under the additional hypothesis that the group has rank 3 . It is proven that if a projective plane $\mathscr{P}$ of order $n>2$ has a rank 3 collineation group then $\mathscr{P}$ is nondesarguesian and either (i) $n$ is odd and $n=m^{4}$, or (ii) $n$ is even and $n=m^{2}$ with $m=0(\bmod 4)$.

One of the older problems in the theory of projective planes is the following: If a finite projective plane $\mathscr{P}$ has a collineation group $G$ transitive on its points, is the plane desarguesian? So far only under one or more additional assumptions has the answer been shown to be yes. The basis for these results is the theorem, due to Wagner [10], that if the transitive group $G$ contains a central collineation, then $\mathscr{P}$ is desarguesian.

Ostrom and Wagner [8] showed that if the group $G$ is doubly transitive then $\mathscr{P}$ is desarguesian, and $G$ contains all elations of $\mathscr{P}$. Higman and McLaughlin [4] investigated the problem in the case when the group $G$ is transitive on the flags of $\mathscr{P}$. They proved that under certain restrictions on the order of $\mathscr{P}$ the plane is desarguesian. Keiser [6] and Wagner [10] have showned that under restrictions on the order of $\mathscr{P}$ and the order of $G$ the plane is desarguesian.

The rank of a permutation group $G$ transitive on a set $\Omega$ is the number of orbits of $G_{P}, P$ a point of $\Omega$, in $\Omega$. Hence a transitive group $G$ has rank 2 on a set $\Omega$ if and only if $G$ is doubly transitive on $\Omega$. $G$ has rank 3 if and only if for every point $P \in \Omega G_{P}$ has two orbits besides $G_{P}$. Ostrom and Wagner have thus answered the question when the group $G$ has rank 2. It is then natural to ask: If a finite projective plane has a transitive collineation group of rank 3 , is the plane desarguesian?

Investigating this question we have found that a more appropriate question is: Which finite projective planes have rank 3 collineation groups? For we will prove in this article the following

MaIn Theorem. Let $\mathscr{P}$ be a finite projective plane of order $n$ with rank 3 collineation group $G$. Then $n$ satisfies one of the statements:

(i) $n=2$

(ii) $n$ is odd and $n=m^{4}$ 
(iii) $n$ is even and $n=m^{2}$ with $m \equiv 0(\bmod 4)$ Furthermore only in case (i) is $\mathscr{P}$ desarguesian.

The proof consists in showing that $G$ must be a nonsolvable flag-transitive group of even order. It is not known whether $\mathscr{P}$ can actually exist in cases (ii) or (iii). Hence the theorem leads to the following conjecture:

ConjeCture. The only finite projective plane having a rank 3 collineation group is the desarguesian plane of order two.

The desarguesian plane of order two (Fig. 1) has the rank 3 collineation group $G$ generated by the collineations $\sigma=\left(P_{1} P_{7} P_{6} P_{4} P_{3} P_{2} P_{5}\right)$ and $\tau=\left(P_{7} P_{6} P_{3}\right)\left(P_{4} P_{5} P_{2}\right)$. Note that $G$ is solvable, sharply flag-transitive, and has order 21 .

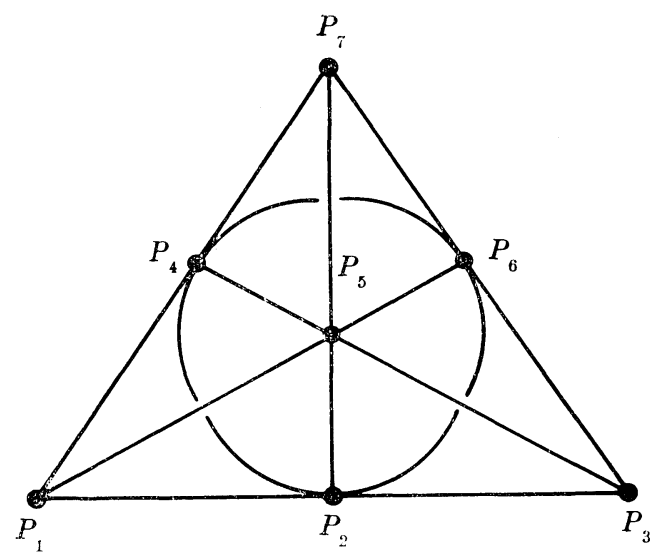

FIGURE 1

We wish to thank Professor Ostrom for many helpful suggestions and for reading a preliminary draft.

2. Definitions and results required later. We assume the reader is familiar with the theory and terminology of projective planes as appears, for example, in Chapters 3-5 of Dembowski [3]. Also a familiarity with the simpler aspects of permutation group theory (as in Chapter 1 of Wielandt [12]) will be assumed.

A transitive collineation group on a projective plane $\mathscr{P}$ is one which is transitive on the points (and hence on the lines by Result II below). A rank 3 collineation group of $\mathscr{P}$ is a transitive collineation group which has rank 3 as a permutation group on the points of $\mathscr{P}$. A flag of $\mathscr{P}$ is an incident point-line pair; i.e., a pair $P, l$ with $P$ a point, $l$ a line, of $\mathscr{P}$ and $P \in l$. A flag-transitive collineation group is one which is transitive on the flags of $\mathscr{P}$.

Use will be made of a number of results concerning permutations 
and collineations. These are listed below for convenience:

Result I (Ostrom [27]; Dembowski [3], p. 214): If $\mathscr{P}$ is a finite projective plane having a collineation group $G$ which is transitive on nonincident point-line pairs, then $G$ is doubly transitive on the points of $\mathscr{P}$.

Result II (Dembowski [2], Hughes [5], Parker [9]): A collineation group of a finite projective plane has equally many point and line orbits.

Result III (Higman and McLaughlin [4]): Let $\mathscr{P}$ be a finite projective plane of order $n$ with a flag-transitive collineation group $G$. If $n$ is odd and not a fourth power, then $\mathscr{P}$ is desarguesian and $G$ contains all elations of $\mathscr{P}$ (See also Dembowski [3], p. 212).

Result IV (Higman and McLaughlin [4]): Let $G$ be a flag-transitive collineation group of a desarguesian projective plane $\mathscr{P}$ of order $n$. $G$ contains all elations of $\mathscr{P}$ with precisely two exceptions:

(i) $n=2$ and $G$ has order 21.

(ii) $n=8$ and $G$ has order 657 .

Result $V$ (Wagner [10]): If $\mathscr{P}$ is a finite projective plane having a transitive collineation group $G$ which contains a nontrivial central collineation, then $\mathscr{P}$ is desarguesian and $G$ contains all elations of $\mathscr{P}$.

Result VI (Keiser [6]): Let $\mathscr{P}$ be a finite projective plane of order $n$ with a transitive collineation group $G$. If $G$ is nonsolvable and if $n=m^{2}$ with $m \equiv 2(\bmod 4)$ or $m \equiv 3(\bmod 4)$, then $\mathscr{P}$ is desarguesian and $G$ contains all the elations of $\mathscr{P}$.

Results VII (Dembowski [3], p. 212): Let $\mathscr{P}$ be a finite projective plane of order $n$. If $G$ is a collineation group which is solvable and primitive on the points of $\mathscr{P}$, then $n^{2}+n+1$ is a prime.

For the next result we note that a permutation group on a set $\Omega$ is regular (sharply transitive) if it is transitive and $G_{P}$ consists only of the identity for each point $P \in \Omega . \quad G$ is a Frobenius group on $\Omega$ if (i) it is transitive, (ii) $G_{P}$ is nontrivial for each point $P \in \Omega$, and (iii) for every two distinct points $P, Q \in \Omega G_{P, Q}$ consists only of the identity.

Result VIII (Wielandt [11], 11.6): A transitive permutation group of prime degree is solvable if and only if it is either regular or a Frobenius group (Due to E. Galois).

3. The investigation. In this section we prove that if a finite projective plane $\mathscr{P}$ has a rank 3 group $G$ of collineations then $G$ is 
flag-transitive on $\mathscr{P}, G$ is nonsolvable (if $n>2$ ), and $\mathscr{P}$ is not desarguesian (if $n>2$ ). We start with

Lemma 1. Let $\mathscr{P}$ be a finite projective plane and $G$ a rank 3 group of collineation of $\mathscr{P}$. Then for every point $P$ of $\mathscr{P}, G_{P}$ permutes the lines through $P$ in one or two orbits.

Proof. $G_{P}$ permutes the points different than $P$ in two orbits $\mathscr{O}_{1}$ and $\mathscr{O}_{2}$. Define the sets $\mathscr{K}_{i}, i=1,2$, by: $\mathscr{K}_{i}$ consists of the lines through $P$ such that $l$ contains at least one point in $\mathscr{O}_{i}$. $G_{P}$ is transitive on $\mathscr{C}_{1}$ and $\mathscr{C}_{2}$, and every line through $P$ is either in $\mathscr{C}_{1}$ or $\mathscr{A}_{2}$. If $\mathscr{C}_{1} \cap \mathscr{C}_{2}$ is empty, then $G_{P}$ permutes the lines through $P$ in two orbits. If $\mathscr{H}_{1} \cap \mathscr{C}_{2}$ is nonempty, then $G_{P}$ is transitive on the lines through $P$.

THEOREM 1. Let $\mathscr{P}$ be a finite projective plane and $G$ a rank 3 group of collineations of $\mathscr{P}$. Then $G_{P}$ is flag-transitive.

Proof. Assume $G$ is not flag-transitive. For every point $P$ of $\mathscr{P}$ $G_{P}$ has 3 point orbits in $\mathscr{P}$. Hence by Result II $G_{P}$ has three line orbits in $\mathscr{P}$. If all the lines through $P$ are in a single orbit under $G_{P}$, then $G$ is flag-transitive contrary to our assumption. Thus by Lemma 1, two of these line orbits consist of the lines through $P$. Thus the third line orbit of $G_{P}$ must consist of all the lines of $\mathscr{P}$ which do not go through $P$. Hence for every point $P, G_{P}$ is transitive on all lines not through $P$.

Let $(P, l)$ be a non-incident point-line pair of $\mathscr{P}$ and $(Q, m)$ another non-incident point-line pair. There exists a collineation $\sigma \in G$ such that $P \sigma=Q$. Let $\bar{l}$ be the image of $l$ under $\sigma . \quad Q \notin \bar{l}$ since $P \notin l$. Then there exists a collineation $\tau \in G_{Q}$ such that $\bar{l} \tau=m$. Then $P \sigma \tau=Q \tau=Q$ and $l \sigma \tau=\bar{l} \tau=m$. This proves $G$ is transitive on non-incident point-line pairs.

Result I implies that $G$ is doubly transitive on the points of $\mathscr{P}$. This is a contradiction since $G$ has rank 3 on the points of $\mathscr{P}$. Thus $G$ is flag-transitive.

Lemma 2. Let $\mathscr{P}$ be a finite projective plane of order $n$ and $G$ a rank 3 group of collineations of $\mathscr{P}$, and let $P$ a point of $\mathscr{P}$.

(i) $G_{P}$ permutes the points not equal to $P$ in two orbits $\mathscr{O}_{1}$ and $\mathcal{O}_{2}$ of lengths $k_{1}(n+1)$ and $k_{2}(n+1)$ respectively, where $k_{1}+k_{2}=n$.

(ii) $G_{P}$ permutes the lines not through $P$ in two orbits $\mathscr{L}_{1}$ and $\mathscr{L}_{2}$ of lengths $s_{1}$ and $s_{2}$ respectively, with $s_{1}+s_{2}=n^{2}$.

Proof. $G_{P}$ is transitive on the lines through $P$. Thus every line 
through $P$ intersects the non-trivial point orbits $\mathscr{O}_{1}$ in the same number of points. Thus $\left|\mathscr{O}_{1}\right|=k_{1}(n+1)$ where $k_{1}$ is the number of points of $\mathscr{O}_{1}$ on a line $l$ through $P$. Similarly $\left|\mathscr{O}_{2}\right|=k_{2}(n+1), k_{2}$ the number of points of $\mathscr{O}_{2}$ on a line $l$ through $P$. This proves (i) since for every line $l$ through $P$, a point $\neq P$ on $l$ is either in $\mathscr{O}_{1}$ or $\mathscr{O}_{2}$.

$G_{P}$ has three point orbits. Hence it has three line orbits (Result II). One of these consists of the lines through $P$. The other two line orbits are made up of the lines not through $P$ and there are $n^{2}$ such lines. This gives (ii).

Lemma 3. Let $\mathscr{P}$ be a finite projective plane of order $n$ and $G$ a rank 3 group of collineations of $\mathscr{P}$. If $G$ is solvable, then

(i) $n^{2}+n+1$ is a prime;

(ii) $G$ acts as a Frobenius group on $\mathscr{P}$;

(iii) $n$ is even, $|G|=1 / 2\left(n^{2}+n+1\right) n(n+1)$, and $\left|G_{P}\right|=1 / 2 n(n+1)$. If $|G|$ is odd, then we also have

(iv) $n=2 m, m$ odd.

Proof. Since $G$ is flag-transitive, $G$ is primitive on the points of $\mathscr{P}$ (Dembowski [3], p. 212). By Result VII $n^{2}+n+1$ is a prime. Result VIII implies that $G$ is either regular on $\mathscr{P}$ or it is a Frobenius group on $\mathscr{P}$. Since $G$ is clearly not regular it must be a Frobenius group on $\mathscr{P}$. This proves (i) and (ii).

By Lemma 2 we have for every point $P \in \mathscr{P}$

$$
\left|G_{P}\right|=k_{1}(n+1)\left|G_{P, Q}\right|=k_{2}(n+1)\left|G_{P, R}\right|,
$$

where $Q \in \mathcal{O}_{1}$ and $R \in \mathcal{O}_{2}$, and $k_{1}+k_{2}=n$. But $G$ a Frobenius group on $\mathscr{P}$ implies $\left|G_{P, Q}\right|=1=\left|G_{P, R}\right|$. Hence $\left|G_{P}\right|=k_{1}(n+1)=k_{2}(n+1)$ and thus $k_{1}=k_{2}=n / 2$. This implies $n$ is even since $k_{1}$ is an integer, and we have $|G|=1 / 2\left(n^{2}+n+1\right)(n(n+1))$. This proves (iii).

If $|G|$ is odd, then $n / 2$ is odd and this proves (iv).

Lemma 4. Let $\mathscr{P}$ be a finite projective plane of order $n$ with a rank 3 group of collineations. If $n>2$, then $|G|$ is even.

Proof. Assume $|G|$ is odd. Then $G$ is solvable (by the FeitThompson theorem) and the previous lemma implies $n^{2}+n+1$ is a prime, $n=2 m$ with $m$ odd, and $|G|=\left(n^{2}+n+1\right) m(n+1)$. Also for each point $P, G_{P}$ has two line orbits $\mathscr{L}_{1}$ and $\mathscr{L}_{2}$ of lengths $s_{1}$ and $s_{2}$ respectively with

$$
s_{1}+s_{2}=n^{2}=4 m^{2}
$$

(Lemma 2). Since $\left|G_{P}\right|=m(n+1)=m(2 m+1)$, we have

$$
s_{i} \mid m(2 m+1), \quad i=1,2 .
$$


Let $s=$ g.c.d. $\left(s_{1}, s_{2}\right)$ and define integers $t_{1}$ and $t_{2}$ by

$$
s_{i}=s t_{i}, \quad i=1,2 .
$$

Then g.c.d. $\left(t_{1}, t_{2}\right)=1$ and $s\left(t_{1}+t_{2}\right)=4 m^{2}$ (by (1)). Since $s$ is odd (for otherwise $\left|G_{P}\right|$ is even), $s \mid m^{2}$. Since $s \mid s_{1}$ (2) implies $s \mid m$ since g.c.d. $(m, 2 m+1)$. Hence

$$
m=s u
$$

for some integer $u$, and

$$
t_{1}+t_{2}=4 s u^{2} \text {. }
$$

If $v=$ g.c.d. $\left(t_{1}, \mathrm{~s}\right)>1$, then $v \mid t_{2}-a$ contradiction to the fact that g.c.d. $\left(t_{1}, t_{2}\right)=1$. If $w=$ g.c.d. $\left(t_{1}, u\right)>1$, then $w \mid t_{2^{-}}$again a contradiction. Hence $1=$ g.c.d. $\left(t_{1}, s\right)=$ g.c.d. $\left(t_{1}, u\right)$, which implies g.c.d. $\left(t_{1}, m\right)=1$. Similarly g.c.d. $\left(t_{2}, m\right)=1$ and thus

$$
t_{i} \mid 2 m+1, \quad i=1,2 .
$$

Then we have, using (5)

$$
2(2 m+1) \geqq t_{1}+t_{2}=4 s u^{2} .
$$

Applying (4) we get

$$
1 \geqq 2 s u(u-1) \text {. }
$$

Therefore $u=1$ and

$$
m=s \text {. }
$$

But then

$$
t_{1}+t_{2}=4 m \text {. }
$$

If $t_{1}=t_{2}$, then $t_{1}=2 m$ and this contradicts (6). Thus without loss of generality we may assume $t_{1}<t_{2}$. Then from (8) we get $t_{1}<2 m$, $t_{2}>2 m$ and this implies $t_{2}=2 m+1$ (by (6)) and $t_{1}=2 m-1$ (by (8)). Hence $2 m-1 \mid 2 m+1$, which implies $m=1, n=2$. This proves the lemma.

REMARK. The example at the end of $\S 1$ shows that if $n=2|G|$ can be odd.

By combining Lemma 3 and Lemma 4 we can show that the rank 3 group $G$ is nonsolvable if $n>2$ :

THEOREM 2. Let $\mathscr{P}$ be a finite projective plane of order $n$ with a rank 3 group $G$ of collineations. If $n>2$, then $G$ is nonsolvable. 
Proof. Assume $G$ is solvable and $n>2$. By Lemma 3(ii) $G$ acts as a Frobenius group on $\mathscr{P}$. Lemma 4 implies $|G|$ is even. Hence $G$ has an element $\sigma$ of order 2. Either $\sigma$ is a central collineation or it fixes a subplane of $\mathscr{P}$ pointwise (Baer [1]). In both cases $\sigma$ fixes more than two points. But $G_{P, Q}$ consists only of the identity for every two distinct points $P, Q$ of $\mathscr{P}$. This gives a contradiction. Therefore $G$ is nonsolvable if $n>2$.

Our last result in this section shows that $\mathscr{P}$ is desarguesian only when $n=2$.

THEOREM 3. Let $\mathscr{P}$ be a finite projective plane with a rank 3 group $G$ of collineations. $\mathscr{P}$ is desarguesian if and only if $n=2$.

Proof. Assume $\mathscr{P}$ is desarguesian. By Theorem $1 G$ is flagtransitive. $G$ cannot contain all the elations of $\mathscr{P}$. For the group $H$ generated by the elations of $\mathscr{P}$ is doubly transitive on the points of $\mathscr{P}$. $H$ a subgroup of $G$ implies $G$ is doubly transitive on the points of $\mathscr{P}$-again contradicting the fact that $G$ has rank 3 on the points of $\mathscr{P}$. By Result IV either $n=2$ and $G$ has order 21, or $n=8$ and $G$ has order 657 . But the second case cannot occur since $n>2$ implies $|G|$ is even (Lemma 4). Thus $n=2$ and $G$ has order 21 .

Conversely if $n=2$, then $\mathscr{P}$ is desarguesian, and the example at the end of $\S 1$ shows that in this case a rank 3 group does occur.

4. Proof of the main theorem. We now prove the main theorem stated in $\S 1$. Assume $n$ is odd. If $n$ is not a fourth power, then Theorem 1 and Result III implies that $\mathscr{P}$ is desarguesian and $G$ contains all elations of $\mathscr{P}$. But by Theorem 3 this is impossible.

Assume $n$ is even and $n>2$. Lemma $4|G|$ is even. If $n$ is not a square, then an element in $G$ of order 2 must be an elation (Baer [1]) and $\mathscr{P}$ is desarguesian by Result V. This contradicts Theorem 3. Hence $n$ is a square. If $n=m^{2}$ with $m \equiv 2(\bmod 4)$, then $\mathscr{P}$ is desarguesian by Result VI since $G$ is nonsolvable (Corollary 2.1). This contradicts Theorem 3 again. The proof of the main theorem is complete.

\section{REFERENCES}

1. R. Baer, Projectivities with fixed points on every line of the plane, Bull. Amer. Math. Soc., 52 (1946), 273-286.

2. P. Dembowski, Verallgemeinerungen von Transitivitätsklassen endlicher projektiver Ebenen, Math. Z., 69 (1958), 59-89.

3. - Finite Geometries, Springer-Verlag, Berlin-Heidelberg, 1968.

4. D. G. Higman and J. E. McLaughlin, Geometric ABA-groups, Ill. J. Math., 5 (1961), 382-397. 
5. D. R. Hughes, Collineations and generalized incidence matrices, Trans. Amer. Math. Soc., 86 (1957), 284-296.

6. V. H. Keiser, Finite projective planes with non-solvable transitive collineation groups, Amer. Math. Monthly, 74 (1967), 556-559.

7. T. G. Ostrom, Dual transitivity in finite projective planes, Proc. Amer. Math. Soc., 9 (1958), 55-56.

8. T. G. Ostrom and A. Wagner, On projective and affine planes with transitive collineation groups, Math. Z., 71 (1959), 186-199.

9. E. T. Parker, On collineations of symmetric designs, Proc. Amer. Math., Soc., 8 (1957), 350-351.

10. A. Wagner, On Perspectivities of finite projective planes, Math. Z., 71 (1959), 113123.

11. H. Wielandt, Finite permutation groups, Acedemic Press, New York and London, 1964.

Received January 12, 1971. This work was partially supported by NSF Contract No. GP. 17461.

WASHINGTON STATE UNIVERSITY 


\section{PACIFIC JOURNAL OF MATHEMATICS}

\section{EDITORS}

H. SAMELSON

Stanford University

Stanford, California 94305

C. R. HobBY

University of Washington

Seattle, Washington 98105
J. DugundjI

Department of Mathematics

University of Southern California

Los Angeles, California 90007

RICHARD ARENS

University of California

Los Angeles, California 90024

\section{ASSOCIATE EDITORS}

E. F. BECKENBACH

B. H. NeUMaNN

F. WOLF

K. YoshidA

\section{SUPPORTING INSTITUTIONS}

UNIVERSITY OF BRITISH COLUMBIA

CALIFORNIA INSTITUTE OF TECHNOLOGY

UNIVERSITY OF CALIFORNIA

MONTANA STATE UNIVERSITY

UNIVERSITY OF NEVADA

NEW MEXICO STATE UNIVERSITY

OREGON STATE UNIVERSITY

UNIVERSITY OF OREGON

OSAKA UNIVERSITY
UNIVERSITY OF SOUTHERN CALIFORNIA STANFORD UNIVERSITY

UNIVERSITY OF TOKYO

UNIVERSITY OF UTAH

WASHINGTON STATE UNIVERSITY UNIVERSITY OF WASHINGTON

$\stackrel{*}{*} \stackrel{*}{*} \stackrel{*}{*}$ AMERICAN MATHEMATICAL SOCIETY
NAVAL WEAPONS CENTER 


\section{Pacific Journal of Mathematics}

Vol. 39 , No. 1

May, 1971

Charles A. Akemann, A Gelfand representation theory for $C^{*}$-algebras ....

Sorrell Berman, Spectral theory for a first-order symmetric system of

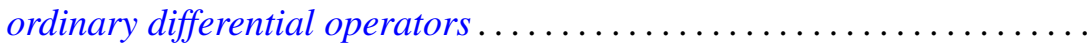

Robert L. Bernhardt, III, On splitting in hereditary torsion theories ........

J. L. Brenner, Geršgorin theorems, regularity theorems, and bounds for determinants of partitioned matrices. II. Some determinantal identities ..........................................

Robert Morgan Brooks, On representing $F^{*}$-algebras .............. 51

Lawrence Gerald Brown, Extensions of topological groups........... 71

Arnold Barry Calica, Reversible homeomorphisms of the real line ........ 79

J. T. Chambers and Shinnosuke Oharu, Semi-groups of local Lipschitzians in

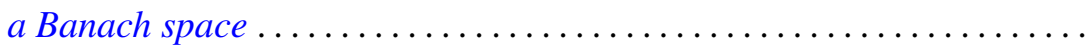

Thomas J. Cheatham, Finite dimensional torsion free rings .............

Byron C. Drachman and David Paul Kraines, A duality between

transpotence elements and Massey products ...................

Richard D. Duncan, Integral representation of excessive functions of a

Markov process ......................................

George A. Elliott, An extension of some results of Takesaki in the reduction

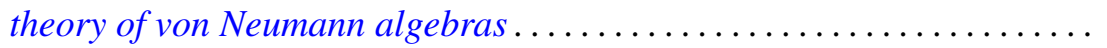

Peter C. Fishburn and Joel Spencer, Directed graphs as unions of partial

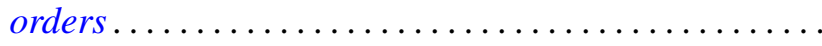

Howard Edwin Gorman, Zero divisors in differential rings ...

Maurice Heins, A note on the Löwner differential equations...

Louis Melvin Herman, Semi-orthogonality in Rickart rings. .

David Jacobson and Kenneth S. Williams, On the solution of linear G.C.D.

equations

Michael Joseph Kallaher, On rank 3 projective planes ... . .

Donald Paul Minassian, On solvable $O^{*}$-groups ...........

Nils Øvrelid, Generators of the maximal ideals of $A(\bar{D})$

Mohan S. Putcha and Julian Weissglass, A semilattice decomposition into

semigroups having at most one idempotent ............

Robert Raphael, Rings of quotients and $\pi$-regularity ....

J. A. Siddiqi, Infinite matrices summing every almost periodic sequence. .

Raymond Earl Smithson, Uniform convergence for multifunctions ...

Thomas Paul Whaley, Mulitplicity type and congruence relations in

universal algebras...

Roger Allen Wiegand, Globalization theorems for locally finitely generated modules... 